\title{
Acknowledgement to Reviewers of Buildings in 2019
}

\section{Buildings Editorial Office}

MDPI, St. Alban-Anlage 66, 4052 Basel, Switzerland

Published: 20 January 2020

The editorial team greatly appreciates the reviewers who have dedicated their considerable time and expertise to the journal's rigorous editorial process over the past 12 months, regardless of whether the papers are finally published or not. In 2019, a total of 239 papers were published in the journal, with a median time to first decision of 14 days and a median time from submission to publication of 36 days. The editors would like to express their sincere gratitude to the following reviewers for their generous contribution in 2019:

Abdelhamid, Tariq Sami

Abdollahnejad, Zahra

Acampa, Giovanna

Adekunle, Timothy Olukunle

Adhikary, Suman Kumar

Ahn, Jun

Akoğlu, Goze

Aletta, Francesco

Ali Ahmed, Awadelrahman

Mohamedelsadig

Ali, Babkir

Ali, Md. Hasan

Alkaff, Saqaff Ahmed Abdulrahman

Al-Kheetan, Mazen

Al-Kodmany, Kheir

Al-Obaidi, Karam

Alonso, Alicia

Al-Rahmani, Ahmed

Altan, Hasim

Alves, Fernando Brandão

Amado, Miguel

Amasyali, Kadir

Amiri, Leyla

Amirkhani, Mehdi

Anastasiu, Livia

Angrecka, Sabina

Antuchevičienė, Jurgita

Anysz, Hubert

Arafa, Ahmed

Argenziano, Pasquale

Armstrong, Paul

Asaee, Rasoul

Aslani, Farhad

Atanasoae, Pavel
Atazadeh, Behnam

Azcarate-Aguerre, Juan Francisco

Baglivo, Cristina

Bąkowski, Jarosław

Balocco, Carla

Banaitis, Audrius

Banica, Alexandru

Banionis, Karolis

Bao, Yi

Barbaresi, Alberto

Barreca, Francesco

Barrios-Padura, Angela

Basińska, Małgorzata

Bastas, Ali

Beach, Thomas H.

Bednarz, Łukasz

Bedon, Chiara

Bellia, Laura

Belloni, Elisa

Bellos, Evangelos

Belussi, Lorenzo

Benni, Stefano

Bento, Rita

Berardi, Umberto

Betti, Michele

Beyabanaki, S. Amir Reza

Bienvenido-Huertas, David

Blecich, Paolo

Boanca, Paunita

Brambilla, Arianna

Braulio-Gonzalo, Marta

Briga-Sá, Ana

Brzyski, Przemysław

Burrascano, Pietro 
Bus, Agnieszka

Caggiano, Antonio

Campana, Pietro

Caniato, Marco

Cannistraro, Mauro

Cardaci, Alessio

Cardone, Sergio

Car-Pusic, Diana

Casado, Alejandro

Cascardi, Alessio

Castaldo, Paolo

Čekon, Miroslav

Cerezo-Narváez, Alberto

Cerro-Prada, Elena

Chan, Daniel W.M.

Chan, Rengie

Charvátová, Hana

Chatzidimitriou, Angeliki

Chen, Ke

Chen, Kian Wee

Chen, Shang-Yuan

Chen, Yan

Cheng, Huanyu

Cheung, Angus K.F.

Chokhachian, Ata

Chuang, Tzu-Yi

Cirella, Giuseppe T.

Coch, Helena

Coletti, Chiara

Conceição, Eusébio Z.E.

Conte, Emilia

Coppola, Luigi

Cortiços, Nuno D.

Costanzo, Vincenzo

Croce, Pietro

Cui, Borui

Czarnecki, Sławomir

Czarnigowska, Agata

D’Adamo, Idiano

Da Silva, Pedro Raposeiro

Da Silva, Sandra Monteiro

Dąbrowski, Mariusz

Dadzie, John

Damialis, Athanasios

Dan, Maria Bostenaru

D'Andrea, Antonio

Dastgerdi, Ahmadreza Shirvani

De La Flor, Francisco José Sanchez

De Paola, Pierfrancesco

De Rubeis, Tullio

De Vita, Mariangela

Del Zoppo, Marta
Denisiewicz, Arkadiusz

Denzer, Anthony S.

Deshmukh, Suhrid

Dhulipala, Somayajulu

Diab, Youssef

Dimyadi, Johannes

Doan, Dat

Domingo-Irigoyen, Silvia

Domínguez-Amarillo, Samuel

Doroudchi, Elahe

Doroudiani, Saeed

Du, Jia-Chong

Durdyev, Serdar

D'Urso, Sebastiano

Dzwierzynska, Jolanta

Elena, Lucchi

Elhattab, Ahmed

Escamilla, Edwin Zea

Estêvão, João M.C.

Estokova, Adriana

Evangelista, Ana Catarina Jorge

Eyssautier, Stéphanie

Fabbri, Kristian

Fabrizio, Enrico

Fangrat, Jadwiga

Fantozzi, Fabio

Farzad, Mahsa

Fatiguso, Fabio

Fayaz, Muhammad

Felseghi, Raluca-Andreea

Feng, Kelvin

Fernández-Agüera, Jessica

Ferrero, Marco

Figueiredo, Carlos

Fikfak, Alenka

Florence, Lequien

Flores, Marta Roig

Fokaides, Paris

Fonseca, Ana

Foraboschi, Paolo

Foti, Giandomenico

Fraddosio, Aguinaldo

Fujii, Kenji

Fujita, Kohei

Futcher, Julie

Gadea, Jose Maria

Galao, Oscar

Galić, Mario

Gallo, Paola

Galvão, João Rafael

Ganah, Abdulkadir

García Calvo, José Luis 
García, Jair E.

Garrido-Velarde, Jacinto

Gayarre, Fernando Lopez

Ghahari, Seyed Ali

Gheisari, Masoud

Ghosh, Aritra

Giannetti, Niccolò

Giuffrida, Salvatore

Giurca, Ioan

Gładyszewska-Fiedoruk, Katarzyna

Goker, Parisa

Gołaszewski, Jacek

Golewski, Grzegorz Ludwik

Gómez-Soberón, Jose Manuel

Goodman, Allen C.

Górecki, Jarosław

Grygierek, Joanna

Habert, Guillaume

Hall, Monika

Hamdy, Mohamed

Hanif, Asad

Hassanain, Mohammad A.

Hayashi, Fumitaka

Hejna, Aleksander

Henriques, Carla Oliveira

Hernandez, Mark

Hilloulin, Benoît

Hirano, Yujiro

Hird, Myra J.

Ibrahim, Nadine

Ihara, Takeshi

Inard, Christian

Inzerillo, Laura

Iringova, Agnes

Isaac, Shabtai

Isufi, Brisid

Iyer-Raniga, Usha

Jacobs, David E.

Jang, Sung-Hwan

Javed, Arshad

Jechow, Andreas

Jensen, Per Anker

Jiang, Ruinian

Jiménez, Javier Martínez

Jimenez-Alonso, Javier Fernando

Jimenez-Bescos, Carlos

Jobst, Markus

Ju, Bu-Seog

Kahle, Eckhard

Kairytė, Agnè

Kalinowska-Wichrowska, Katarzyna

Kang, James
Kapliński, Oleg

Karczmarczyk, Agnieszka

Kassai, Miklos

Katunský, Dušan

Katzer, Jacek

Khanolkar, Gauri

Khatib, Jamal

Khosravani, Mohammad Reza

Kim, Gyuyong

Kim, Hyun Woo

Kim, HyungKyoo

Kim, Junkyeong

Kim, Sun-Sook

Kim, Taehoon

Kim, Young Sik

Klimeš, Lubomír

Koda, Eugeniusz

Köhler, Manfred

Kong, Xiangxiong

Konstantakopoulou, Foteini

Kontoni, Denise-Penelope N.

Koulinas, Georgios K.

Koutamanis, Alexander

Kovalevsky, Andrei V.

Krajewski, Piotr

Kraus, Michal

Krawczyk, Łukasz

Król, Małgorzata

Krzywon, Rafal

Kujawa, Joanna

Kula, Krzysztof

Kuo, Wen-Ten

Kurata, Narito

La Russa, Mauro Francesco

Lage-Cal, Susana

Lanzinha, Joao

Lanzinha, João

Łapka, Piotr

Laurini, Eleonora

Leccese, Fabio

Leccese, Francesco

Lee, Byung Hee

Lee, Jae Min

Lee, Kyungsun

Lekidis, Vassilios

Leśniak, Agnieszka

Levacher, Daniel

Li, Hong Xian

Liberato, Ferrara

Lignola, Gian Piero

Lill, Irene

Lim, Jae-Han 
Lima, Carmine

Lin, Zhibin

Lino, Barbara

Littmann, Klaus

Liu, Hexu

Liu, Xin

Lo, L. James

Lombardi, Mara

López-Ochoa, Luis María

Loss, Cristiano

Louis, Joseph

Lu, Ming

Lu, Peiwen

Lucchi, Elena

Maalouf, Chadi

Mager, Tino

Mainka, Anna

Malaiškienė, Jurgita

Man Li, Rita Yi

Mander, John

Mangalathu, Sujith

Manganelli, Benedetto

Marinakis, Vangelis

Marović, Ivan

Marques, Rui

Martín-Gómez, César

Martins, Ana Maria Tavares

Mateo-Garcia, Monica

Mazzeo, Domenico

Medved, Sergej

Melgar, Sergio Gómez

Meresi, Aik.

Michaud, Gilbert

Milovanovic, Bojan

Mistrick, Richard G.

Mojtahedi, Mohammad

Moldovan, Macedon

Monica, Luigi

Montuori, Rosario

Mora, Rodrigo

Morea, Donato

Moretti, Laura

Morillas, Leandro

Munari, Marco

Muscio, Alberto

Mwasha, Abrahams

Nagai, Kohei

Nagy, Balázs

Nardi, Iole

Narloch, Piotr

Naser, Mohannad Z.

Nastasi, Benedetto
Nawari, Nawari

Neri, Manuela

Neves, Rui

Newton, Clare

Nik, Vahid

Nikolic, Jelena

Ninikas, Konstantinos

Nord, Natasa

Nouri, Andre Santos

Nourozi, Behrouz

Nowogonska, Beata

Olbina, Svetlana

Oldfield, Philip

Omer, Siddig Adam

Ostrowski, Krzysztof

Oswald-Tranta, Beate

Ozarisoy, Bertug

Ozkan, Aylin

Pacheco-Torgal, F.

Palella, Boris

Palella, Boris Igor

Palumbo, Elisabetta

Panda, Biranchi

Paolini, Riccardo

Papalou, Angeliki

Paraskevopoulou, Angeliki

Park, Jihyun

Park, Jin Chul

Park, Sangki

Pastor, José L.

Pavlů, Tereza

Payne, Vance William

Pearce, Linda

Peng, Chengzhi

Pereira, Pedro F.

Pereira, Sandra

Pezzuolo, Andrea

Pezzutto, Simon

Piekarczuk, Artur

Pitti, Rostand Moutou

Plebankiewicz, Edyta

Pnevmatikos, Nikos

Poletti, Elisa

Pomares, Jorge C.

Pons, Oriol

Porojan, Liliana

Pour, Mohsen Saffari

Psunder, Igor

Pujadas, Pablo

Puppio, Mario Lucio

Pushkar, Svetlana

Putra, Handi Chandra 
Rad, Aryan Rezaei

Radianti, Jaziar

Radosław, Jasiński

Raheem, Adeeba Abdul

Ramis-Soriano, Jaime

Rao, Ajith

Rebaudengo, Manuela

Renigier-Bilozor, Malgorzata

Resende, Ricardo Pontes

Rey, Juan Rey

Rizo-Maestre, Carlos

Rocchi, Lucia

Rodrigues, Antonio Moret

Rodrigues, Eugénio

Rogers, Judith

Rogulj, Katarina

Romano, Rosa

Rosa, Flavio

Rosenberg, Elissa

Ross, Brandon

Rosso, Federica

Rousakis, Theodoros

Rubio-Bellido, Carlos

Ruggieri, Nicola

Ruiz, Germán Ramos

Russo, Alessio

Russo, Salvatore

Sadar, John

Sajjadian, Masoud

Sakellaris, Ioannis A.

Salimifard, Parichehr

Salman, Ibrahim N. A.

Salom, Iva

Sameer, Husam

Samouh, Hamza

Sampaio, Alcínia Z.

Sandberg, Marcus

Santi, Giovanni

Santos, Ricardo

Santos-Herrero, Jose Maria

Šaparauskas, Jonas

Sapienza, Vincenzo

Sareen, Siddharth

Sargentis, George-Fivos

Sarker, Prabir

Sassani, Alireza

Sayed-Ahmed, Ezzeldin Yazeed

Scamoni, Fabio

Schabowicz, Krzysztof

Scheinherrová, Lenka

Schneider, Sven

Schouenborg, Björn
Scott, Lloyd

Seifan, Mostafa

Sepasgozar, Samad

Sferra, Adriana

Shah, Raj Kapur

Shahnewaz, Md

Shea, Andrew

Shehu, Rafael

Shin, Yoon Hyuk

Shiva, Javad Shafiei

Sigmund, Zvonko

Šipoš, Tanja Kalman

Skiba, Marta

Sližytė, Danutè

Šneideris, Arnoldas

Socha, Tomasz

Song, Yongze

Sousa, Hélder S

Sousa, Hipólito

Spina, Lucia Della

Srinivasan, Ravi

Stec, Agnieszka

Stepinac, Mislav

Streimikiene, Dalia

Strug, Barbara

Strukar, Kristina

Suárez, Rafael

Suchorab, Zbigniew

Šuman, Nataša

Sunindijo, Riza

Suraneni, Prannoy

Suszanowicz, Dariusz

Švajlenka, Jozef

Szafranko, Elżbieta

Szeląg, Maciej

Tagawa, Hiroshi

Tajani, Francesco

Tamas-Gavrea, Daniela-Roxana

Tamošaitienė, Jolanta

Tang, Chao-Wei

Tao, Chengcheng

Taylor, Thomas J.

Teresa, Mangialardi

Torgal, F. Pacheco

Tracz, Tomasz

Trgala, Kamil

Tri Phung, Quoc

Tronchin, Lamberto

Tsai, Kang-Ting

Tsai, Meng-Ting

Tsangouri, Eleni

Tsangrassoulis, Aris 
Tso, Chi Yan

Tubaldi, Enrico

Tulliani, Jean Marc

Tutak, Magdalena

Ukrainczyk, Neven

Ullrich, Torsten

Urbán, Daniel

Uros, Mario

Usman, Muhammad

Valdiserri, Paolo

Valipoor, Shabboo

Varo-Martinez, Marta

Vasconcelos, Francisco

Veintimilla, Salvador García-Ayllón

Verdolotti, Letizia

Vermeir, Gerrit

Verre, Salvatore

Viñas, Carmen

Vlachokostas, Alex

Vogtländer, Joost

Vona, Marco

Vulic, Milivoj

Wahlgren, Paula

Waibel, Christoph

Wakili, Karim

Walker, Andy

Wang, Hongxia

Wang, Hsiang-Chen
Wang, Junjie

Wang, Xiao Yong

Wang, Xiaodong

Wan-Wendner, Roman

Wardeh, George

Webborn, Ellen

Werner-Juszczuk, Anna Justyna

Wright, Laurie

Wrona, Paweł

$\mathrm{Wu}$, Hwai-Chung

Xie, YuLong

$\mathrm{Xu}, \mathrm{Qi}$

Yang, Bin

Yang, Jyh-Bin

Yang, Wei

Yang, Xiaotian

Yau, Yung

Yeon, Jaeheum

Yeon, Kyu-Seok

Yin, Jie

Yu, Fu-wing

Zaki, Syed Ahmed

Zamora-Polo, Francisco

Zani, Giulio

Zavadskas, Edmundas Kazimieras

Zheng, Xiaofeng

Zubizarreta, Mikel

(C) 2020 by the authors. Licensee MDPI, Basel, Switzerland. This article is an open access article distributed under the terms and conditions of the Creative Commons Attribution (CC BY) license (http://creativecommons.org/licenses/by/4.0/). 